\title{
Prevalence of hypertension in Thailand: Hotspot clustering detected by spatial analysis
}

\author{
Wongsa Laohasiriwong, ${ }^{1}$ Nattapong Puttanapong, ${ }^{2}$ Atthawit Singsalasang ${ }^{3}$ \\ ${ }^{1}$ Faculty of Public Health and Research and Training Center for Enhancing Quality of Life for Working \\ Age People, Khon Kaen University, Khon Kaen; ${ }^{2}$ Faculty of Economics, Thammasat University, Bangkok; \\ ${ }^{3}$ Faculty of Public Health, Khon Kaen University, Khon Kaen, Thailand
}

\begin{abstract}
Spatial pattern detection can be a useful tool for understanding the geographical distribution of hypertension (HT). The aim of this study was to apply the technique of local indicators of spatial association statistics to examine the spatial patterns of HT in the 76 provinces of Thailand. Previous studies have demonstrated that socioeconomic status (SES), economic growth, population density and urbanization have effects on the occurrence of disease. Research has suggested that night-time light (NTL) can be used as a proxy for a number of variables, including urbanization, density, economic growth and SES. To date, there has not been any study on spatial patterns of HT and there is no information on how NTL might correlate with HT. Therefore, this study has investigated NTL as a parameter for detection of hotspots of HT in Thailand. It
\end{abstract}

Correspondence: Wongsa Laohasiriwong, Faculty of Public Health, Khon Kaen University, Khon Kaen, 40002, Thailand.

Tel.: +66.087.373.1199 - Fax: +66.43347058.

E-mail: drwongsa@gmail.com

Key words: Hypertension; Local indices of spatial association; Nighttime light; Thailand.

Acknowledgments: the authors are grateful to all of the contributors to this research, especially the National Statistical Office for the data.

Contributions: the authors contributed equally.

Conflict of interest: the authors declare no potential conflict of interest.

Funding: the authors are grateful to the Research and Training Center for Enhancing Quality of Life for Working Age People for the financial support.

Received for publication: 12 July 2017

Revision received: 8 January 2018.

Accepted for publication: 8 January 2018.

CCopyright W. Laohasiriwong et al., 2018

Licensee PAGEPress, Italy

Geospatial Health 2018; 13:608

doi:10.4081/gh.2018.608

This article is distributed under the terms of the Creative Commons Attribution Noncommercial License (CC BY-NC 4.0) which permits any noncommercial use, distribution, and reproduction in any medium, provided the original author(s) and source are credited. was found that HT clusters occurred in Bangkok and in metropolitan areas. In addition, significantly low-rate clusters were seen in some provinces in the Northeast and also in southern provinces. These findings should facilitate control and prevention of HT and, therefore, serve as support for researchers, decision-makers, academics and public health officials to propose more sound and effective strategies for the control of HT in Thailand and elsewhere.

\section{Introduction}

Hypertension (HT) is a non-communicable disease with enhanced morbidity and mortality and whose increasing trend worldwide is seen as a global public health challenge. HT is one of the top modifiable risk factors in the field of cardiovascular disease (CVD) (Kayima et al., 2015). In 2025, according to the World Health Organization (WHO), the number of patients in industrialized countries who will have developed HT is estimated at 1.15 billion (WHO, 2015). Moreover, half of those patients will die from ischemic heart disease and stroke where HT is the root cause (MoPH, 2007; Bharati and Ajayi, 2011; WHO, 2015). In Thailand, for instance, it was found that in 2003, 2008 and 2013, the morbidity rate due to HT per 100,000 people was 389.80 , 860.53 and 1621.72, respectively, indicating an exponential increase in rate $(\mathrm{MoPH}, 2015)$. Many factors are associated with HT, such as age, gender, having a family history of HT, and alcohol consumption (Oliveira et al., 2014; Wei et al., 2015), in addition to overweight/obesity, smoking (Wei et al., 2015), physical inactivity, high dietary salt intake (Chongthawonsatid, 2013), stress (Lwin et al., 2011), education, occupation (Lwin et al., 2011; Wei et al., 2015), economic status, income and social status (Puavilai et al., 2011). These factors are known to contribute to the risk of HT and cerebrovascular disease. In addition, some previous studies also found differences in regional location to be associated with HT (Satipunyalert, 2014; Wei et al., 2015; Abebe et al., 2015). Residential areas may have influenced health behaviour in terms of lifestyle, social well-being and urbanization (Bharati and Ajayi, 2011). Studies concerning Mae Hong Son Province in Thailand (Satipunyalert, 2014), Dehui City in Jilin Province in China (Wei et al., 2015) and the Northwest of Ethiopia (Abebe et al., 2015) report a significantly higher tendency for HT in urban areas or cities in contrast to that in rural areas.

Many epidemiological studies indicate that HT occurs in different geographic and metropolitan regions (Satipunyalert, 2014; Wei et al., 2015; Abebe et al., 2015). Geographic information systems (GIS), together with other instruments used for geographic pattern analysis (George et al., 2013) are important tools for geo- 
graphic analysis. Anselin (1995) has demonstrated the usefulness of applying local indicators of spatial association (LISA) in epidemiological settings. For instance, Rosana Rosseto de Oliveira et al. (2012) studied infant mortality in three cities in Paraná state, Brazil using LISA for the analysis of spatial distribution and autocorrelation. Wang et al. (2016) analyzed spatial autocorrelation analysis and hotspot analysis of the HIV/AIDS epidemic applying LISA.Night-time light measurements (NTLs) (Mellander et al., 2015; Laohasiriwong et al., 2017) have been used to represent economic growth, population density and urbanization. NTL has also been used in public health and epidemiological studies in recent years, e.g., Laohasiriwong et al. (2017) utilized NTL to assess economic growth, urbanization and health care facility coverage. Areas with a prevalence of chronic respiratory disease (CRD) also experience increased light resulting in a correlation with NTL and socioeconomics (Laohasiriwong et al., 2017). NTLs may serve as a new tool for specifying disease hotspots by representing population and industrial growth which typically contribute to epidemics. In urban areas with migration, NTLs can indicate where populations are clustering, through the expansion and increased brightness of the illuminated areas (Henderson et al., 2012). NTLs can also indicate fluctuations in population density, which affect epidemic risk and can therefore replace current methods of outbreak surveillance (Princeton University, 2011).

In Thailand, the government has used various tools to assess HT. However, spatial clustering of the disease, particularly at the national level, has rarely been applied to investigate characteristics of HT clusters in terms of geography. In addition, there has been limited use of socioeconomic status (SES) indicators, especially NTLs, in the epidemiology of HT. The aims of this study were to examine the spatial clustering of HT in Thailand and to clarify if there is a correlation between NTLs and HT. The findings should provide data to support interventions in specific high-rate areas. Academics, researchers, decision makers and public health personnel will be able to use the results to develop new strategies for HT control and prevention at the regional level.

\section{Materials and Methods}

\section{Study area}

Thailand is an upper-middle-income country in Southeast Asia with a population of approximately 68 million as of 2017 . The latitude and longitude coordinates are $15.8700^{\circ} \mathrm{N}, 100.9925^{\circ} \mathrm{E}$. The capital and largest city is Bangkok. Thailand shares a total of 4863 $\mathrm{km}$ of border with neighbouring countries, i.e., Myanmar and Laos to the North, the Gulf of Thailand and Malaysia to the South, Laos and Cambodia to the East, and the Andaman Sea and the southern extremity of Myanmar to the West (Figure 1). With the addition of a new province, Bung Kan, in 2011, Thailand is divided into 76 provinces (Figure 1).

\section{Data sources}

This study used data from the National Socioeconomics Survey (NSS), a cross-sectional study conducted by the National Statistical Office (NSO) in 2005, 2006 and 2007. It represents the latest nationwide surveys incorporating detailed questionnaires regarding health status and chronic diseases. The surveys used stratified two-stage random sampling, firstly selecting provinces and then selecting blocks/villages. In this way, a nationally representative sample was selected to respond to a structured questionnaire for all 76 provinces in Thailand. There were 76 strata altogether and each stratum was divided into two parts according to the type of local administration, namely, municipal and nonmunicipal areas. Selection of primary sampling, i.e., the selection of blocks/villages, was performed separately and independently in each part by using a probability proportional to the total number of households in that block or village. In the second step, the selection of secondary sampling units, i.e., private households, was based on using a systematic method in each type of local administration (details of this sampling are available at http://web.nso.go.th/survey/house_seco/meth.pdf. The questionnaires collected information under seven categories consisting of education, health care, employment, income, finances, migration and attitudes/opinions. The outcome, defined as having HT diagnosed previously by a physician, was based on a blood pressure reading of 140/90 $\mathrm{mmHg}$ or higher and with the individual being continuously treated for HT. A total of 16,306, 16,539 and 16,488 participants, who met the inclusion criteria of Thai nationality and being aged 15 years or over, were included in the analysis for each year. The NTL data for Thailand used in this study were extracted from the global stable lights imagery of 2005-2007 collected by the Operational Linescan System (OLS) sensor onboard satellites

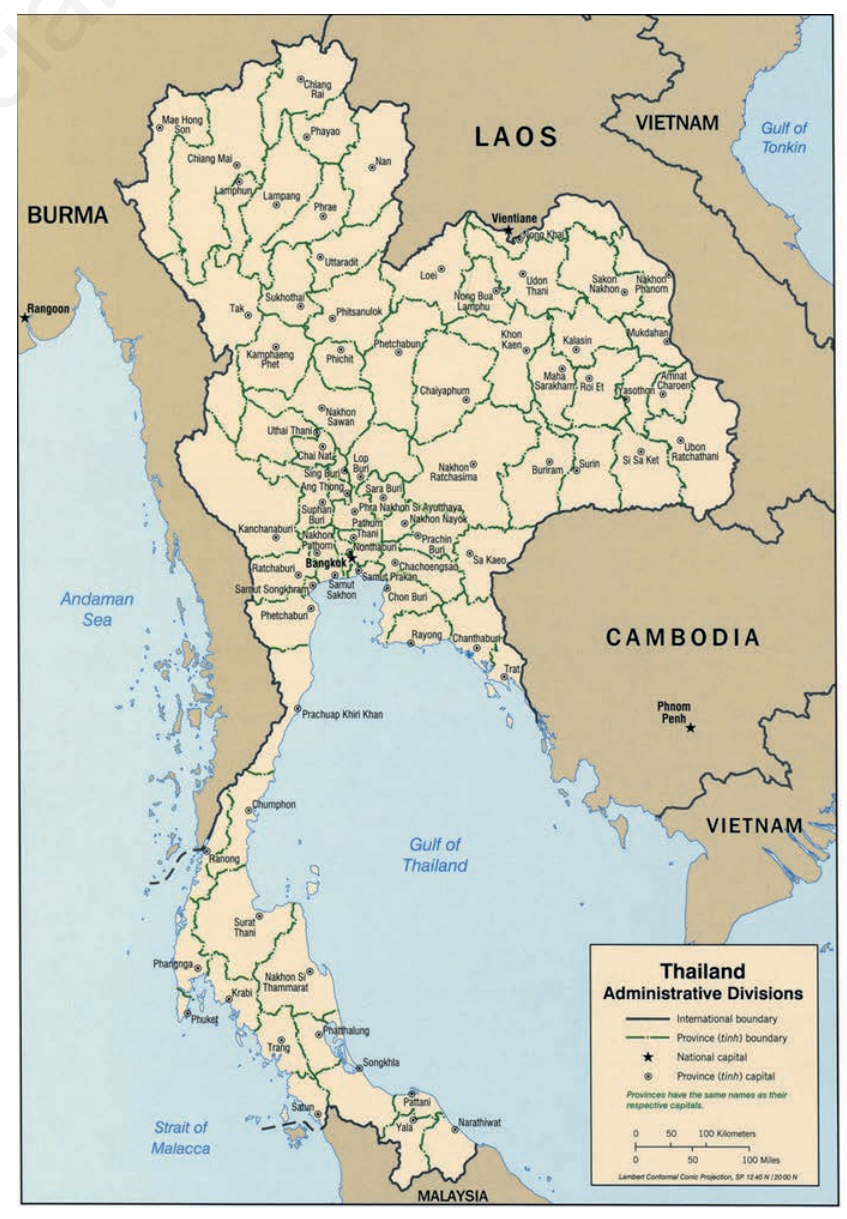

Figure 1. Map of Thailand and its provinces. 
F15 (F152005, F152006 and F152007) and F16 (F162005, F162006 and F162007) of the Defence Meteorological Satellite Program (DMSP), USA (Figure 2).

\section{Spatial pattern detection}

The detection of HT spatial patterns was based on localized detection of prevalent spatial patterns. For exploratory spatial data analysis we used the open GIS software Quantum GIS (QGIS) introduced by Steiniger and Hunter (2013), together with GeoDa (https://geodacenter.github.io) to determine measures of spatial autocorrelation analysis (Anselin, 2006), and Stata version 10.0 (Stata Corp, College Station, TX, USA) to calculate the prevalence of HT, and for correlation analysis. The prevalence of HT for each province was calculated as the total number of HT participants in that province (the numerator) divided by the total number of participants in that province (denominator). Thus, the spatial distribution of the prevalence of HT could be visualized. To conduct a spatial autocorrelation analysis on the spatial distribution, the geographic patterns and clusters were measured using LISA (Anselin, 1995). The province-level layers of polygons and points were matched to the prevalence of HT. The spatial distribution of the prevalence of HT was obtained from the province-level polygon map, which included the latitudes and longitudes for each province. The calculated prevalence of HT was further visualized with province-level layers on the polygon map and labelled with the administrative code for each province. Exploratory spatial pattern analysis was also used to determine the distribution patterns for the prevalence of HT for each year of data.

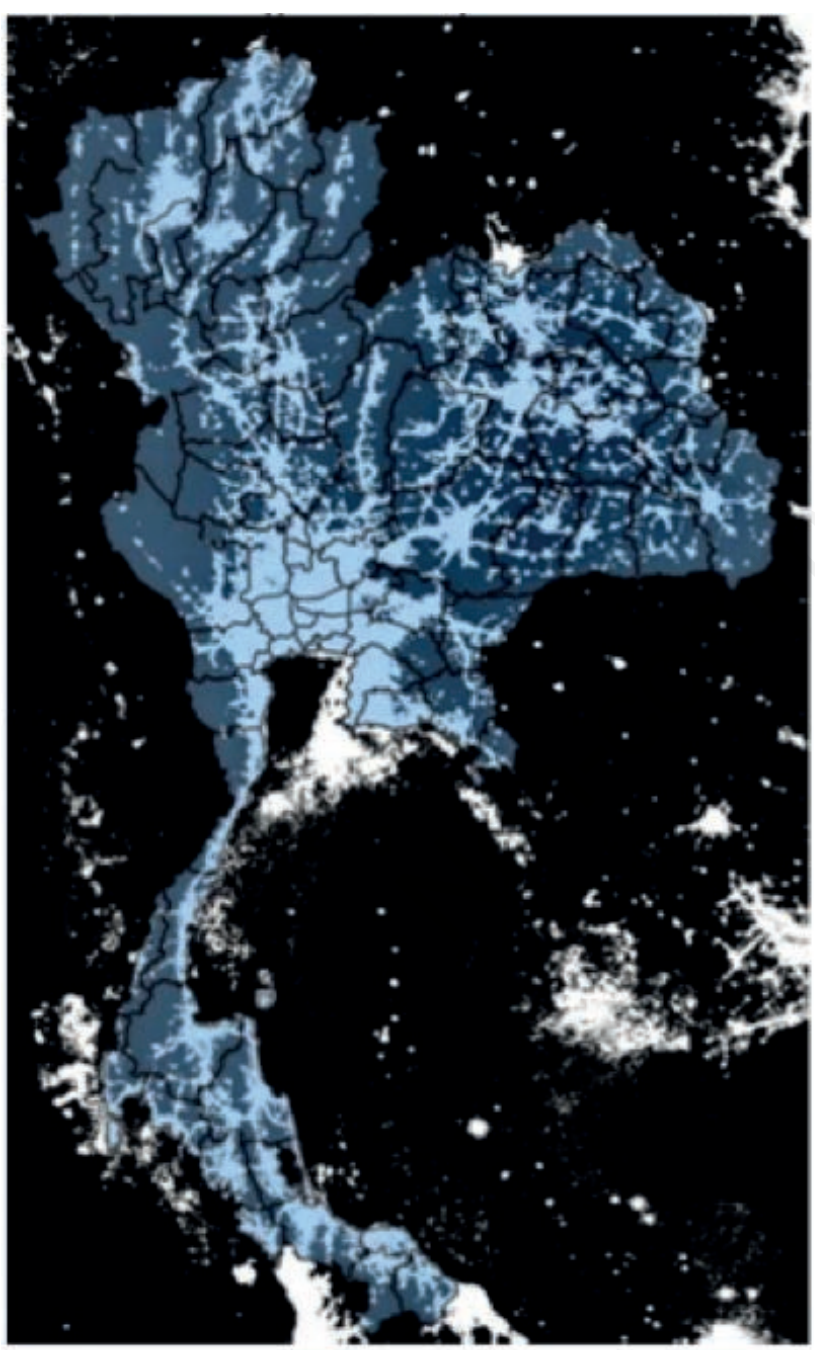

Source: DMSP/OLS data and authors computed

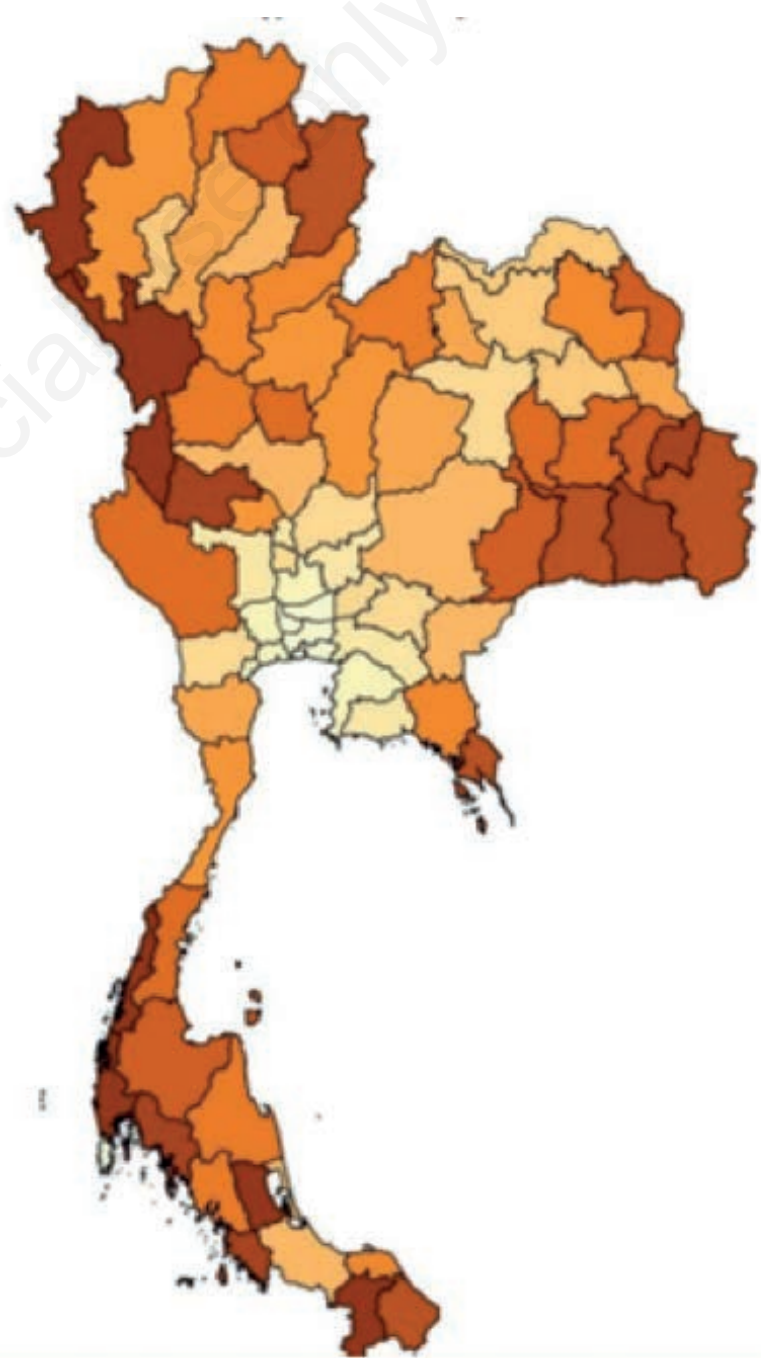

Source: Authors' computation Note: Bright color indicates the high magnitude of nighttime light index

Figure 2. Raw data for night-time light from Defence Meteorological Satellite Program/Operational Linescan System (DMSP/OLS). 


\section{Statistical approach}

\section{Local indications of spatial autocorrelation}

Anselin (1995) made a significant advance within areal analysis by defining a class of LISA as a tool for exploratory spatial data analysis. In the present study, LISA was used to analyze the spatial distribution, and the GeoDa program was used to reveal the prevalence of HT. In addition, the QGIS program was used to prepare data before input into the GeoDa program. The initial data, where HT was prevalent in the province locations, were combined with the NTL data. The results from QGIS were saved as a shape file. Univariate spatial pattern analysis used HT clusters analysis, and bivariate spatial pattern analysis used HT clusters with NTL data analysis. The shape file data were then entered into the GeoDa program for spatial distribution analysis. The weighting matrix with knearest neighbours was 3 and the significance level (P-value) of 0.05 was chosen. The results generated a Moran's $I$ index (Anselin, 1995; Anselin L et al., 2006) and a coloured map. The value of the local Moran's $I$ ranges from +1 (indicating high-high or low-low clusters) through 0 (indicating random pattern) to -1 (indicating high-low or low-high outliers) (Barrell J and Grant J, 2013; Wang et al., 2016). The maps were represented by a five-colour scheme, with high-high assigned as red (hotspot), low-low as blue (coldspot), low-high as light blue, high-low as pink and random patterns shown as white (Anselin, 2003).

In this study, the local Moran's I index was used for cluster feature analysis. There were five possible results when computed by LISA: high-high, low-low, low-high and high-low were significant areas and one area (random pattern) was not significant. Hotspots were deemed to have a high prevalence of HT, whereas coldspots denoted low prevalence. Mixtures of high and low scores in neighbouring areas were considered as spatial outliers. The meanings of the parameters in both equations were the same as those described for the local Moran's I formula. In addition, we considered NTL for bivariate spatial pattern analysis, which may be important for local spatial pattern detection of the HT prevalence. A Pearson's correlation was run to assess the relationship between NTL and the prevalence of HT in all provinces.

\section{Results}

\section{Correlations between night-time light and hypertension}

The provincial Pearson's correlation studies of NTL and HT prevalence showed moderate positive correlations in $2005(0.533$; 95\% CI: 0.346-0.679; $\mathrm{P}<0.001), 2006$ (0.510; 95\% CI: 0.318 $0.661 ; \mathrm{P}<0.001)$ and 2007 (0.490; 95\% CI: 0.294-0.646; $\mathrm{P}<0.001)$, as indicated by the similar Pearson's correlation values in the three years (Table 1).

\section{Local spatial patterns}

The spatial pattern technique indicated that the results were similar in all three years. The results showed that clusters of HT occurred in Bangkok and other metropolitan areas. In addition, significant low-rate clusters were noted in some provinces in the northeast and in southern regional provinces. The univariate Moran's I scatter for annualized prevalence of HT in 2005, 2006 and 2007 showed a slight positive spatial autocorrelation, the Moran's $I$ values being $0.201,0.151$ and 0.186 , respectively; moreover, the values were significant ( 0.05 significance level), which indicated clustering. Moreover, there were hotspots (high-high) in Bangkok and in the adjoining provinces of Nakhon Pathom, Nonthaburi, Pathum Thani and Samut Prakan for all three years, while coldspot (low-low) clusters were located in some provinces in the north-eastern and southern regions (Figures 3 and 4).

Table 1. The correlation between night-time light and hypertension for 2005, 2006 and 2007.

\begin{tabular}{cccc} 
Year & Correlation level & $95 \%$ CI & P-value \\
2005 & 0.533 & $0.346-0.679$ & $<0.001$ \\
2006 & 0.510 & $0.318-0.661$ & $<0.001$ \\
\hline 2007 & 0.490 & $0.294-0.646$ & $<0.001$ \\
\hline
\end{tabular}
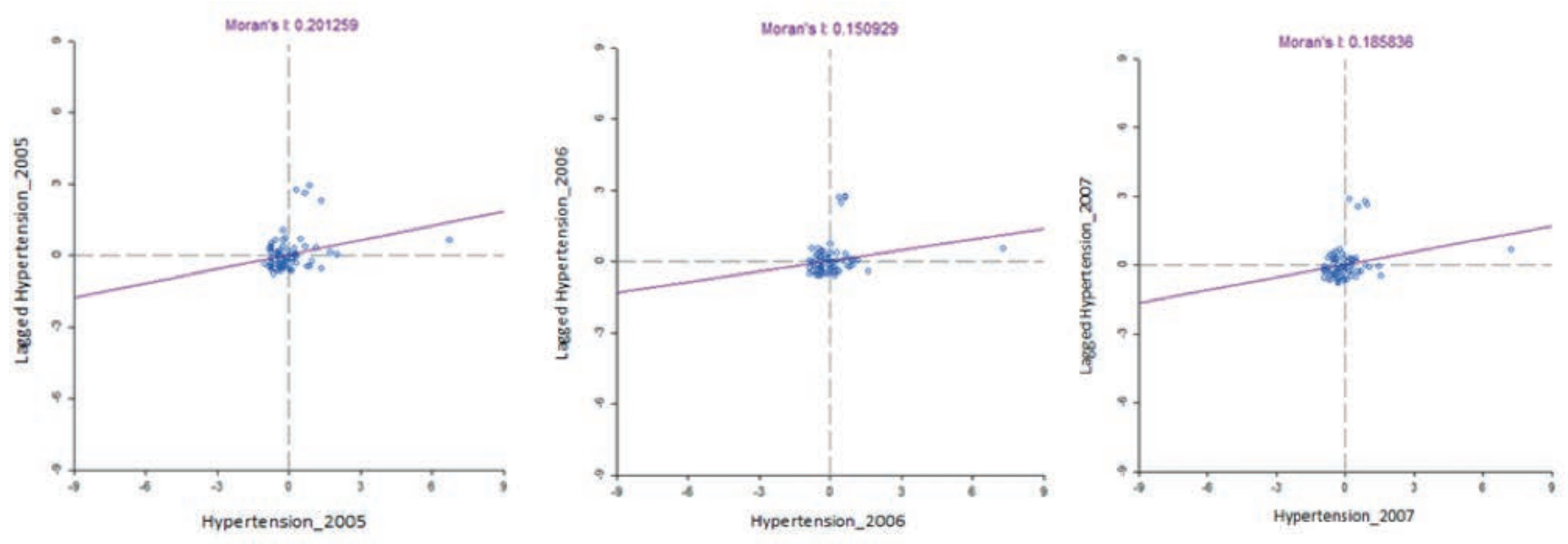

Figure 3. Moran's I scatter plot matrix (univariate) for prevalence of hypertension for 2005-2007. 
The results showed a significant correlation between NTL and HT. This would suggest that NTL in areas with high population densities can be used to define the spatial extent of development of HT. The results indicated a positive spatial autocorrelation, the Moran's $I$ values for 2005, 2006 and 2007 being significant at the 0.05 significance level with values of $0.488,0.473$ and 0.499 , respectively. For all three years, the identified hotspot clusters were located in the Bangkok and metropolitan areas of Nakhon Pathom, Nonthaburi, Pathum Thani and Samut Prakan, while coldspot clusters occurred in some provinces in the northeastern and southern regions; these findings (Figures 5 and 6) are similar to the results for univariate analysis (Figure 4).
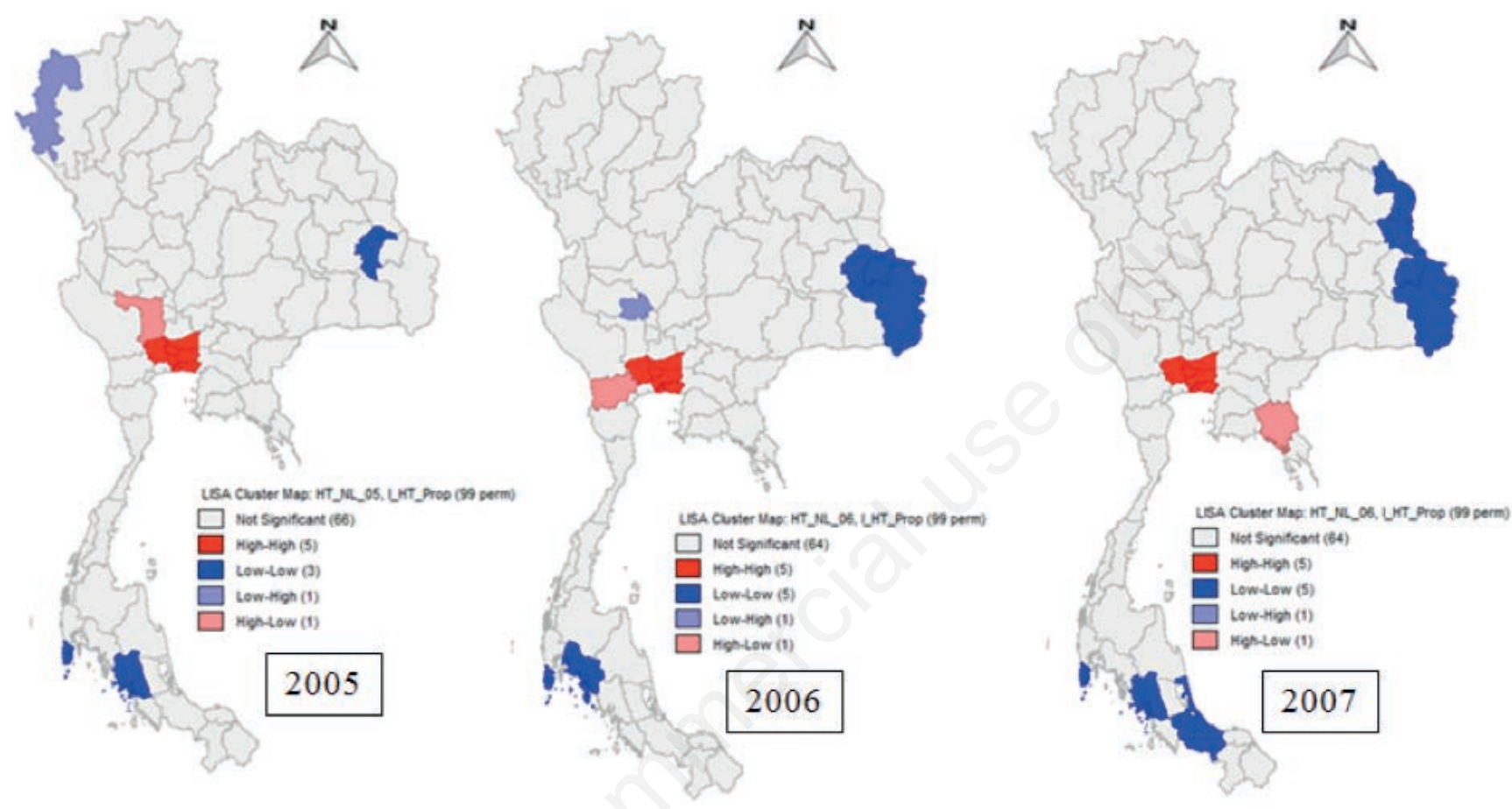

Figure 4. LISA (univariate) map for prevalence of hypertension for 2005-2007.
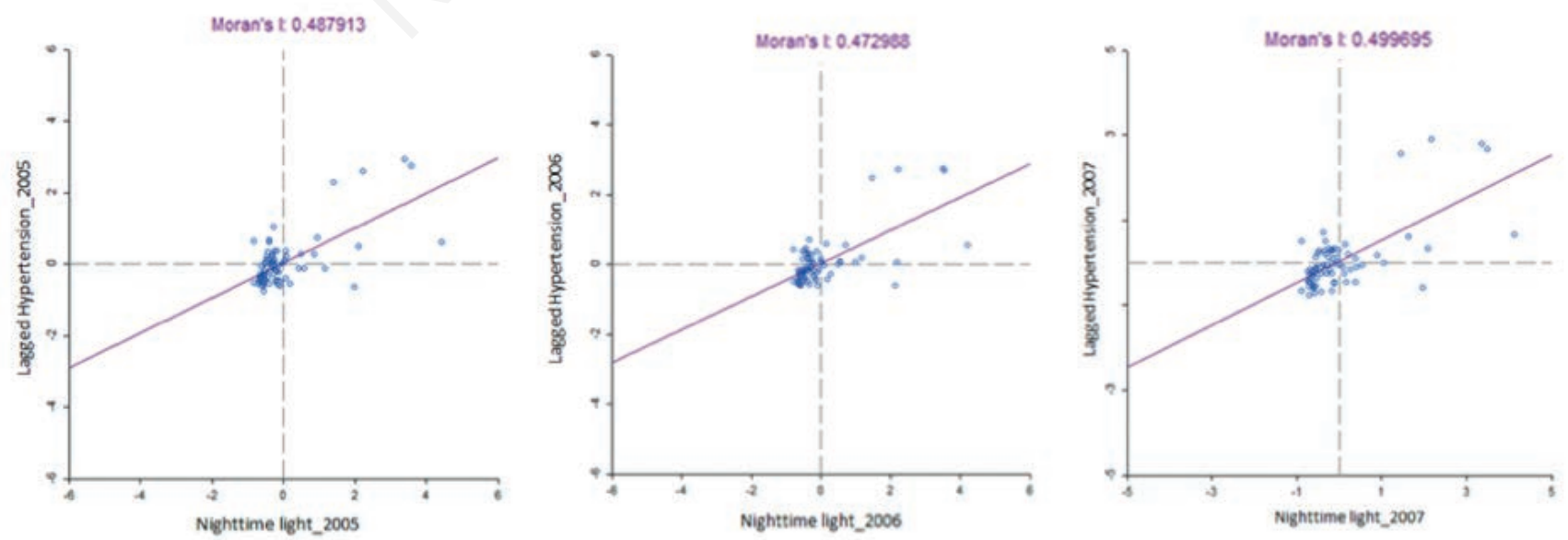

Figure 5. Moran's I scatter plot matrix (Bivariate: night-time light and hypertension) for prevalence of hypertension for 2005,2006 and 2007. 
In general, as seen in Figures 3-6, the LISA statistical results concurred with Bangkok and metropolitan areas having the greatest clustering of HT.

\section{Discussion}

A correlation between NTL and the prevalence of HT was established in this study. The findings showed significant correlation between NTL and HT at the province level. The NTL data may represent differences in factors such as geography, economic growth, urbanization, and healthcare facilities. Such correlations may be used to assess economic growth in the provinces as prposed by Mellander et al. (2015). In this study, the geographical disparities indicated differences in individual and socioeconomic factors, including population density, urbanization, residential area per capita and human behavior or lifestyle; and these factors were found to be determinants in the spatial dynamics of HT in Thailand as discussed by Satipunyalert (2014). The relationship of the NTL data to population size was similar to that of the HT data, a fact that could indicate NTL representing factors affected differently, such as various environmental determinants of illness or health problems given the disparities in socioeconomic status, social determinants and lifestyle mentioned by other authors (Puavilai et al., 2011; Wei et al., 2015; Lwin et al., 2015). The recent use of NTL for the analysis or estimation of spatial inequalities has highlighted many health problems which were found to be dependent on spatial differences or spatial inequalities (Chaiwat, 2016). The present study is also consistent with the findings of Bharti et al. (2011) and after the correlation has been confirmed at the provincial level throughout Thailand, it is suggested that remote sensing focused on NTL may be used in public health studies. The NTL data would represent factors such as population density, urban areas, economic status and social environment, risk factors already implicated in health and disease problems for the area (Bharati and Ajayi, 2011).

The NTL data represented population size in the areas investigated and this coincided with highly significant hotpots for HT. Comparison of the three maps in each year yielded similar findings in terms of the location of HT hotspots on each map. The findings indicated that both the univariate and the bivariate methods detected high incidences of HT in Bangkok and the metropolitan areas. At the same time, low rates of HT were indicated in some provinces in the north-eastern and southern regions of Thailand. The LISA analysis established five major cities (Bangkok, Nakhon Pathom, Nonthaburi, Pathum Thani and Samut Prakan) as being at the core of the high-rate cluster. LISA proved effective in spatial pattern analysis in that high-rate and low-rate HT regions were accurately classified. Although there could be additional factors controlling the prevalence of HT in these five cities compared to other metropolitan regions, the hotspot areas did experience dynamic changes in urban populations and in the concentration of industries. The identification of hotspots specifies priority areas for

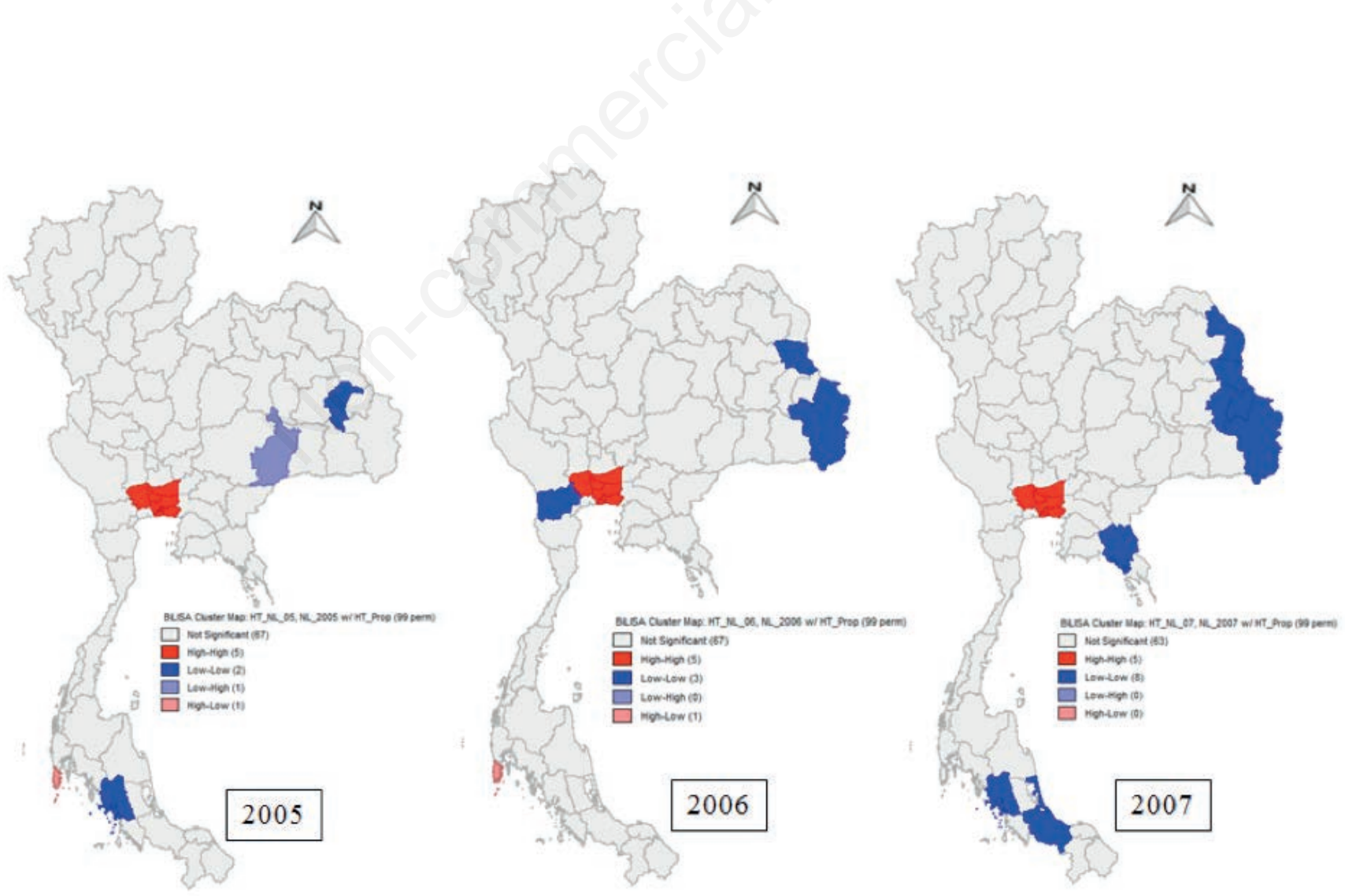

Figure 6. LISA (Bivariate: night-time light and hypertension) map for prevalence of hypertension for 2005-2007. 
HT control and prevention supporting local health centres in their administration of health promotion and prevention measures appropriate to the lifestyles of people in the community. The findings therefore indicate that the application of multiple GIS methods is an effective approach for identifying clusters of HT prevalence at the province level. Moreover, no single technique was considered the best; the most appropriate technique(s) for spatial cluster analysis should be selected because each approach has its advantages and disadvantages (Sasson et al., 2012).

The results show that the prevalence of HT was highest in Bangkok and the metropolitan areas. It is well known that the environment can influence health. Many people who have lived previously in other regions of Thailand have migrated to the cities for work, thus increasing urbanization and industrialization. The social environment affects the lifestyle, which represents an important risk factors for HT (Bharati and Ajayi, 2011); other factors are lack of physical activity (Chongthawonsatid, 2013), alcohol consumption (Oliveira et al., 2014; Wei et al., 2015), stress (Lwin et al., 2011), and socioeconomic disparities such as level of education, occupation, and income (Wei et al., 2015; Lwin et al., 2011; Puavilai et al., 2011). In contrast, the north-eastern and southern regions differ from the metropolitan areas as these regions have a more rural environment and retain a richer and more traditional culture. Most of the population work in the agricultural sector, engage in more outdoor activity, and spend much less time travelling to work. Given these circumstances, the prevalence of HT is low.

\section{Conclusions}

The LISA statistic can be used in spatial pattern analysis to examine the prevalence of disease related to clusters. LISA can also identify the focus of high- and low-incidence clusters of HT. Spatial analysis is particularly appropriate for recognizing clusters of HT because HT is associated with environmental factors, such as location, e.g., urban or rural area, social environment and lifestyle. The hotspot regions of HT display significant spatial risk factors for socioeconomic disparity, such as the level of monthly household income, education and geographic location, all of which may be represented by NTL. The findings showed significant correlation between NTL and HT at the provincial level. These findings can be used in health policy and planning by public health personnel and the health delivery service. With respect to the hotspot areas, the health and human development sectors should be given priority in terms of proactive strategies and actions which should include health promotion and HT prevention, proactive case reporting and prompt treatment and rehabilitation for those individuals suffering from $\mathrm{HT}$ and its complications. Using the map as a guide to specify risk factors in the regions could contribute to more efficient preventive and control programs for HT with relevance to the social environmental context.

\section{References}

Abebe SM, Berhane Y, Worku A, Getachew A, 2015. Prevalence and associated factors of hypertension: a cross sectional community based study in northwest Ethiopia. PloS one 10:512-21.

Anselin L, 1995. Local Indicators of Spatial Association (LISA). Geogr Anal 27:93-115.
Anselin L, 2003. An introduction to spatial autocorrelation analysis with GeoDa. Spatial Analysis Laboratory, University of Illinois. Champagne-Urbana, Illinois.

Anselin L, Syabri I, Kho Y, 2006. GeoDa: an introduction to spatial data analysis. Geogr Anal 38:5-22.

Barrell J, Grant J, 2013. Detecting hot and cold spots in a seagrass landscape using local indicators of spatial association. Landscape Ecol 28:2005-18.

Bharati VM, Ajayi KS, 2011. Hypertension in the developing world: challenges and opportunities. Am J Kidney Dis 55:5908.

Bharti N, Tatem AJ, Ferrari MJ, Grais RF, Djibo A, Grenfell BT, 2011. Explaining seasonal fluctuations of measles in Niger using nighttime lights imagery. Science 334:1424-7.

Chaiwat T, 2016. Night lights, economic growth, and spatial inequality of Thailand. Puey Ungphakorn Institute for Economic Research, Thailand.

Chongthawonsatid S, 2013. Demographic factors and health care behavior of hypertension disease in Thailand. SUSTJ 9:9-16.

George J, Po-Huang C, Tyler S, Rachel B, William K, Bereketab L, Hui-Chen T, Christina W, 2013. Use of GIS Mapping as a public health tool: from cholera to cancer. Health Serv Insights 6:111-6.

Henderson JV, Storeygard A, Weil DN, 2012. Measuring economic growth from outer space. Am Econ Rev 102:994-1028.

Kayima J, Nankabirwa J, Sinabulya I, Nakibuuka J, Zhu X, Rahman M, Longenecker C, Katamba A, Mayanja-Kizza H, Kamya M, 2015. Determinants of hypertension in a young adult Ugandan population in epidemiological transition-the MEPI-CVD survey. BMC Pub Health 15:830-42.

Laohasiriwong W, Puttanapong N, Luenam A, 2017. A comparison of spatial heterogeneity with local cluster detection methods for chronic respiratory diseases in Thailand. F1000 Res 6:1819.

Lwin MMK, Tassanee S, Oranut P, Chaweewon B, 2011. Risk factors for hypertension among rural Thais. Southeast Asian J Trop Med Public Health 42: 208-17.

Mellander C, Lobo J, Stolarick K, Matheson Z, 2015. Night-time light data: a good proxy measure for economic activity? Plos One 10:e0139779.

Ministry of Public Health, 2007. Thailand Health Profile 20052006. Bureau of Policy and Strategy Ministry of Public Health Press, Nonthaburi, Thailand.

Ministry of Public Health, 2015. Health report 2014-2015. Available from: http://203.157.10.11/report/std18report/ rep_R01_preht_thailand56.php? region $=0$

Oliveira GF, Oliveira TR, Ikejiri AT, Andraus MP, Galvao TF, Silva MT, Pereira MG, 2014. Prevalence of hypertension and associated factors in an indigenous community of central Brazil: a population-based study. PloS One 9:862-78.

Puavilai W, Laorugpongse D, Prompongsa S, Sutheerapatranont S, Siriwiwattnakul N, Muthapongthavorn N, Srilert P, Jakpechyothin J, 2011. Prevalence and some important risk factors of hypertension in Ban Paew District. J Med Assoc Thai 94:1069-76.

Princeton University, 2011. Nighttime images help track disease from the sky. Available from: https://www.sciencedaily. com/releases/2011/12/111208145343.htm. Accessed: January 2017.

de Oliveira RR, da Costa JR, Mathias TA, 2012. Spatial distribution and autocorrelation of infant mortality for three cities in 
Paraná state, Brazil. Geospat Health 6:257-62.

Sasson C, Cudnik MT, Nassel A, Semple H, Magid DJ, Sayre M, Keseg D, Haukoos JS, Warden CR, 2012. Identifying high-risk geographic areas for cardiac arrest using three methods for cluster analysis. Acad Emerg Med 19:139-46.

Satipunyalert H, 2014. Factors Related to hypertension of people who live in the area of Thai-Myanmar borderline, Mae Hong Son Province. Master of Public Health. Chiang Mai University, Thailand.

Steiniger S, Hunter AJ, 2013. The 2012 free and open source GIS software map: A guide to facilitate research, development, and adoption. Comput Environ Urban Syst 39:136-150.

Wang Y, Yang Y, Shi X, Mao S, Shi N, Hui X, 2016. The spatial distribution pattern of human immunodeficiency virus/ acquired immune deficiency syndrome in China. Geospat Health 11:414.

Wei Q, Sun J, Huang J, Zhou HY, 2015. Prevalence of hypertension and associated risk factors in Dehui city of Jilin province in China. J Hum Hypertens 29:64-8.

World Health Organization, 2015. New data highlight increases in hypertension, diabetes incidence 2014. Available from: http://www.who.int/mediacentre/en/ 